\title{
New remarks on the linear constraint self-dual boson and Wess-Zumino terms
}

\author{
Everton M. C. Abreu* and Alvaro de Souza Dutra ${ }^{\dagger}$ \\ Departamento de Física e Química, Universidade Estadual Paulista, Avenida Ariberto Pereira da Cunha 333, \\ Guaratinguetá, 12516-410, São Paulo, SP, Brazil \\ (Received 25 April 2001; published 16 August 2001)
}

\begin{abstract}
In this work we prove in a precise way that the soldering formalism can be applied to the Srivastava chiral boson (SCB), in contradiction with some results appearing in the literature. We promote a canonical transformation that shows directly that the SCB is composed of two Floreanini-Jackiw particles with the same chirality in which the spectrum is a vacuumlike one. As another conflicting result, we prove that a Wess-Zumino (WZ) term used in the literature consists of a scalar field, once again denying the assertion that the WZ term adds a new degree of freedom to the SCB theory in order to modify the physics of the system.
\end{abstract}

DOI: 10.1103/PhysRevD.64.065003

PACS number(s): 11.10.Ef, 04.65.+e

\section{INTRODUCTION}

The research in chiral bosonization began many years back with the seminal paper by Siegel [1]. Floreanini and Jackiw later offered some different solutions to the problem of a single self-dual field [2], proposing a nonanomalous model. The study of chiral bosons has blossomed thanks to advances in some string theories [3] and in the construction of interesting theoretical models [4]. These also play an important role in studies of the quantum Hall effect [5]. The introduction of a soliton field as a charge-creating field obeying one additional equation of motion leads to a bosonization rule [6]. Stone [7] showed that the method of a coadjoint orbit [8], when applied to a representation of a group associated with a single affine Kac-Moody algebra, generates an action for the chiral Wess-Zumino-Witten model [9], a nonAbelian generalization of the Floreanini-Jackiw (FJ) model.

A self-dual field in two dimensions is a scalar field which satisfies the self-dual constraint (self-dual condition) $\left(\eta^{\mu \nu}\right.$ $\left.+\epsilon^{\mu \nu}\right) \partial_{\nu} \phi=0$ or $\dot{\phi}=\phi^{\prime}$, where an overdot means time derivation and a prime space derivation. In the formulation of Floreanini and Jackiw [2], the space derivative of the field instead of the field itself satisfies the self-dual condition, i.e., $\left(\partial_{0}-\partial_{1}\right) \partial_{1} \phi=0$, and the field violates the microcausality postulate [10].

Trying to overcome these difficulties, Srivastava [11] introduced an auxiliary vector field $\lambda_{\mu}$ coupled with a linear constraint, and constructed a Lorentz-invariant Lagrangian for a scalar self-dual field. Although Harada [12] and Girotti et al. [13] pointed out consistency problems with the Srivastava model at the quantum level, the linear formulation strictly describes a chiral boson from the point of view of equations of motion at the classical level. Some methods were used to quantize the theory [14]. The extension to $D$ $=6$ was accomplished in Ref. [15], as well as its supersymmetric case [16].

On the other hand, the concept of soldering [7,17] has proved extremely useful in different contexts. The soldering

\footnotetext{
*Email address: everton@feg.unesp.br

${ }^{\dagger}$ Email address: dutra@feg.unesp.br
}

formalism essentially combines two distinct Lagrangians manifesting dual aspects of some symmetry, to yield a new Lagrangian which is divested of, or rather hides, that symmetry. The quantum interference effects, whether constructive or destructive, among the dual aspects of symmetry, are thereby captured through this mechanism [18]. The formalism introduced by Stone was recently interpreted as a method of dynamical mass generation [18]. This technique parallels a similar phenomenon in two-dimensional field theory known as the Schwinger mechanism [19] that results from the interference between right and left massless selfdual modes of a chiral Schwinger model [20] of opposite chiralities [18].

Furthermore, an important ingredient in the study of such kinds of systems are the so called Wess-Zumino (WZ) terms [21], which are introduced in the theory in order to recover the gauge invariance [22]. In Ref. [23], a way was proposed to derive the WZ counterterm. It was based on the generalized Hamiltonian formalism of Batalin and Fradkin [24], who suggested a kind of quantization procedure for secondclass constraint systems to which the anomalous gauge theory belongs $[22,25]$. The final action obtained, dependent on an arbitrary parameter, was constructed in order to become Srivastava model gauge invariant. The Lorentz invariance requirement fixed the parameter in two possible values which generate two possible WZ terms. The result, with one of the WZ terms, after a kind of chiral decomposition, was that the Srivastava chiral boson (SCB) spectrum is composed of two opposite FJ chiral bosons, similarly to what happens with the minimal chiral Schwinger model [26]. The conclusion, however, was that the WZ term thus obtained has added a new physical degree of freedom, an antichiral boson, to the spectrum, and therefore would change the self-dual field into a massless scalar. In addition, in another similar paper, Miao and Chen [27] asserted that it is impossible to apply the soldering formalism $[7,17]$ to solder two opposite chiral aspects of the model proposed by Srivastava, as successfully accomplished in the Siegel and Floreanini-Jackiw theories [28]. It was pointed out that the method was invalid in the linear formulation because of the inequivalence of Srivastava's, Siegel's, and FJ's models. Hence, to promote the fusion, a chiral counterterm [23] was constructed for the linear formulation of the chiral bosons. This counterterm was the 
same Wess-Zumino term mentioned above.

In this work we demonstrate that both conclusions are not really true. We successfully apply the soldering formalism, and show that the interference on shell of two SCB results in a massless scalar field. As another result, we perform essentially a canonical transformation (CT) [29,30] (as a special case of a CT, we use dynamical decomposition [31], which promotes a separation of a chiral theory into dynamical and symmetry parts), and the outcome shows, in an exact way, that the spectrum is already composed of two FJ chiral bosons with the same chirality, confirming the well-known result that the SCB has two degrees of freedom thanks to the linear constraint structure [32]. In addition, we show that the WZ term introduced in Ref. [23] is in fact a scalar field, i.e., it is composed of two FJ bosons with opposite chiralities. Thus, it is obvious that the WZ terms naturally introduce these particles, since the spectrum of the SCB is a vacuumlike one [33].

In Sec. II we briefly review the soldering formalism. In Sec. III we carry out the soldering of two SCB models. The dynamical decomposition of the theory and a discussion of the WZ term are accomplished in Sec. IV. Conclusions are made in Sec. V.

\section{SOLDERING FORMALISM}

In this section we will basically follow Ref. [34] and briefly review the method of soldering two opposite chiral versions of a given theory. For more details, the interested reader can see Refs. [18,35,36].

The basic idea of the soldering procedure is to raise a global Noether symmetry of the self-dual and anti-self-dual constituents into a local constituent, but for an effective composite system, consisting of the dual components and an interference term. An iterative Noether procedure was adopted [34] to lift the global symmetries. Therefore, we assume that the symmetries in question are described by the local actions $S_{ \pm}\left(\phi_{ \pm}^{\eta}\right)$, invariant under a global multiparametric transformation

$$
\delta \phi_{ \pm}^{\eta}=\alpha^{\eta}
$$

$\eta$ represents the tensorial character of the basic fields in the dual actions $S_{ \pm}$and, for notational simplicity, will be dropped from now on. As it is well known, we can write

$$
\delta S_{ \pm}=J^{ \pm} \partial_{ \pm} \alpha
$$

where $J^{ \pm}$are the Noether currents.

Now, under local transformations, these actions will not remain invariant, and Noether counterterms will become necessary to reestablish the invariance, along with an appropriate auxiliary field $B^{(N)}$, a so-called soldering field which has no dynamics. Nevertheless we can say that $B^{(N)}$ is an auxiliary field which makes a wider range of gauge-fixing conditions available [17]. In this way, the $N$ action can be written as

$$
S_{ \pm}\left(\phi_{ \pm}\right)^{(0)} \rightarrow S_{ \pm}\left(\phi_{ \pm}\right)^{(N)}=S_{ \pm}\left(\phi_{ \pm}\right)^{(N-1)}-B^{(N)} J_{ \pm}^{(N)} .
$$

Here $J_{ \pm}^{(N)}$ are the $N$-iteration Noether currents. For self-dual and anti-self-dual systems, we have in mind that this iterative gauging procedure is (intentionally) constructed not to produce invariant actions for any finite number of steps. However, if after $N$ repetitions, the noninvariant piece ends up being dependent only on the gauging parameters, but not on the original fields, there will exist the possibility of mutual cancelation if both self- and anti-self-gauged systems are put together. Suppose that, after $N$ repetitions, we arrive at the following simultaneous conditions:

$$
\delta S_{ \pm}\left(\phi_{ \pm}\right)^{(N)} \neq 0 \quad \text { and } \quad \delta S_{B}\left(\phi_{ \pm}\right)=0,
$$

with $S_{B}$ being the so-called soldered action

$$
S_{B}\left(\phi_{ \pm}\right)=S_{+}^{(N)}\left(\phi_{+}\right)+S_{-}^{(N)}\left(\phi_{-}\right)+(\text {contact terms }),
$$

where the contact terms are generally quadratic functions of the soldering fields. Then we can immediately identify the (soldering) interference term as

$$
S_{i n t}=(\text { contact terms })-\sum_{N} B^{(N)} J_{ \pm}^{(N)} .
$$

Incidentally, the auxiliary field $B^{(N)}$ may be eliminated, for instance, through its equations of motion, from the resulting effective action, in favor of the physically relevant degrees of freedom. It is important to note that after the elimination of the soldering fields, the resulting effective action will not depend on either self- or anti-self-dual fields $\phi_{ \pm}$, but only in some collective field, say $\Phi$, defined in terms of the original fields in a (Noether) invariant way:

$$
S_{B}\left(\phi_{ \pm}\right) \rightarrow S_{e f f}(\Phi)
$$

Analyzing in terms of classical degrees of freedom, it is obvious that we have now a larger theory. Once such an effective action has been established, the physical consequences of the soldering are readily obtained by simple inspection.

\section{SOLDERING OF TWO SRIVASTAVA SELF-DUAL BOSONS}

The Srivastava action for a left-moving chiral boson is

$$
\mathcal{L}_{\phi}^{(0)}=\partial_{+} \phi \partial_{-} \phi+\lambda_{+} \partial_{-} \phi
$$

where we have used the light-front variables $\partial_{ \pm}=(1 /$ $\sqrt{2})\left(\partial_{0} \pm \partial_{1}\right)$ and $\lambda_{ \pm}=\lambda_{0} \pm \lambda_{1}$. Following the steps of the soldering formalism studied in Sec. II, we can start by considering the variation of the Lagrangians under the transformations $\delta \phi=\alpha$ and $\delta \lambda_{+}=0$. We will set down only the main steps of the procedure.

In terms of the Noether currents we can construct

$$
\delta \mathcal{L}_{\phi}^{(0)}=J_{\phi}^{\mu} \partial_{\mu} \alpha
$$

where $\mu=+,-, J_{\phi}^{+}=0$, and $J_{\phi}^{-}=2 \partial_{+} \phi+\lambda_{+}$.

The next iteration, as seen in Sec. II, can be performed introducing auxiliary fields, the so-called soldering fields 


$$
\mathcal{L}_{\phi}^{(1)}=\mathcal{L}_{\phi}^{(0)}-B_{\mu} J_{\phi}^{\mu}
$$

and one can easily see that the gauge variation of $\mathcal{L}_{\phi}^{(1)}$ is

$$
\delta \mathcal{L}_{\phi}^{(1)}=-2 B_{-} \delta B_{+},
$$

where we have defined the variation of $B_{ \pm}$as $\delta B_{ \pm}=\partial_{ \pm} \alpha$, and we see that the variation of $\mathcal{L}_{\phi}^{(1)}$ does not depend on $\phi$. This is the signal to begin the process with the other chirality, which is given by

$$
\mathcal{L}_{\rho}^{(0)}=\partial_{+} \rho \partial_{-} \rho+\lambda_{-} \partial_{+} \rho
$$

again, let us construct the basic transformations $\delta \rho=\alpha$ and $\delta \lambda_{-}=0$. Noether's currents are $J_{\rho}^{+}=2 \partial_{+} \rho+\lambda_{-}$and $J_{\rho}^{-}$ $=0$ and the variation of the final iteration is $\delta \mathcal{L}_{\rho}^{(1)}=$ $-2 B_{-} \delta B_{+}$.

Now we can see that the variation of $\mathcal{L}_{\phi, \rho}^{(1)}$ depends neither on $\phi$ nor $\rho$. Hence, as explained above we can construct the final (soldered) Lagrangian as

$$
\begin{aligned}
\mathcal{L}_{T O T} & =\mathcal{L}_{L} \oplus \mathcal{L}_{R} \\
& =\mathcal{L}_{\phi}^{(1)}+\mathcal{L}_{\rho}^{(1)}+B_{+} B_{-} \\
& =\mathcal{L}_{\phi}^{(0)}+\mathcal{L}_{\rho}^{(0)}-B_{+} J^{+}-B_{-} J^{-}+B_{+} B_{-},
\end{aligned}
$$

which remains invariant under the combined symmetry transformations for $(\phi, \rho)$ and $\left(\lambda_{+}, \lambda_{-}\right)$, i.e., $\delta \mathcal{L}_{T O T}=0$.

Following the steps of the algorithm depicted in Sec. II, we have to eliminate the soldering fields, solving their equations of motion, which result in $B_{ \pm}=J^{\mp}$ where $J^{ \pm}=J^{\phi, \rho}$. Substituting this back into Eq. (13) we have the final effective Lagrangian density

$$
\begin{aligned}
\mathcal{L}_{T O T}= & \left(\partial_{-} \phi-\partial_{-} \rho\right)\left(\partial_{+} \phi-\partial_{+} \rho\right)+\lambda_{+}\left(\partial_{-} \phi-\partial_{-} \rho\right) \\
& -\lambda_{-}\left(\partial_{+} \phi-\partial_{+} \rho\right)-\frac{1}{2} \lambda_{+} \lambda_{-} \\
= & \partial_{-} \Phi \partial_{+} \Phi+\lambda_{+} \partial_{-} \Phi-\lambda_{-} \partial_{+} \Phi-\frac{1}{2} \lambda_{+} \lambda_{-} .
\end{aligned}
$$

where the new compound field is defined as $\Phi=\phi-\rho$.

As we can see, we have a second-order term in the Lagrange multipliers. Solving the equations of motion for the multipliers, we obtain that

$$
\lambda_{-}=2 \partial_{-} \Phi \text { and } \quad \lambda_{+}=-2 \partial_{+} \Phi \text {. }
$$

Substituting Eqs. (15) into Eq. (14) we have

$$
\mathcal{L}_{T O T}=-\frac{1}{2} \partial_{\mu} \Phi \partial^{\mu} \Phi
$$

which represents the massless scalar field action.

Hence we have demonstrated in a precise way that it is possible to use the soldering formalism to promote the fusion of two opposite SCB's, in contradiction with the assertion made in Ref. [27]. Finally, one can conclude that, starting from these inconsistent Lagrangian densities, one recovers, in the soldering procedure, a consistent model which is, in fact, a free scalar field. However, this result was not the expected one. We will come back to this issue later.
In Sec. IV we will investigate the spectrum of the Srivastava model, constructing a canonical transformation [30], i.e., using the special case of the dynamical decomposition [31]. The objective is to analyze the result obtained previously by Miao et al. [23] with an alternative construction of the Wess-Zumino term of the Srivastava theory.

\section{DYNAMICAL DECOMPOSITION OF THE SRIVASTAVA MODEL}

In the Hamiltonian formulation, canonical transformations can sometimes be used to decompose a composite Hamiltonian into two distinct pieces. A familiar example [29] is the decomposition of the Hamiltonian of a particle in two dimensions moving in a constant magnetic field and quadratic potential. It can be shown that this Hamiltonian can be separated into two pieces corresponding to the Hamiltonians of two one-dimensional oscillators rotating in clockwise and counterclockwise directions, respectively. Let us now make a canonical transformation analysis of the SCB. In this case, the theory is already a chiral one, and we will promote a dynamical decomposition of it, i.e., the theory will be decomposed into its dynamical and symmetry parts. If the theory is not invariant, the result will show only the dynamics of the system. To perform this we have to make a canonical transformation [30] in Eq. (8), using the Faddeev-Jackiw first-order procedure.

At this point, some interesting comments are in order. The inconsistencies of the SCB model at the quantum level, discussed in some works $[12,13]$, can be verified from another point of view. This is done by comparing the Lagrangian density of the SCB in Minkovisky space, i.e.,

$$
\begin{aligned}
\mathcal{L} & =\frac{1}{2} \partial_{\mu} \phi \partial^{\mu} \phi+\lambda_{\mu}\left(g^{\mu \nu}-\epsilon^{\mu \nu}\right) \partial_{\nu} \phi \\
& =\frac{1}{2}\left(\dot{\phi}^{2}-\phi^{\prime 2}\right)+\lambda\left(\dot{\phi}-\phi^{\prime}\right)
\end{aligned}
$$

where $\lambda=\lambda_{+}$, with that of the bosonized version of the CSM,

$$
\mathcal{L}=\frac{1}{2}\left(\partial_{\mu} \phi\right)^{2}+e\left(g^{\mu \nu}-\epsilon^{\mu \nu}\right) \partial_{\mu} \phi A_{\nu}+\frac{a e^{2}}{2} A_{\mu}^{2}-\frac{1}{4} F_{\mu \nu}^{2},
$$

and to note that the former is in fact a particular case of the latter, where one should take care of the identifications $a$ $=0$ and $A_{\mu} \rightarrow \lambda_{\mu}$, an external field with vanishing field strength. Now one can relate the inconsistency of the SCB to that of the CSM with the regularization ambiguity parameter $a=0$, as shown by Girotti et al. [37]. Now let us resume the discussion of the SCB, performing its dynamical decomposition and then discussing how and why the WZ terms introduced in Ref. [23] recover the SCB's quantum consistency.

The canonical momentum is defined by $\pi=\dot{\phi}+\lambda$. Substituting this back into Eq. (17) to obtain the first-order form, we have

$$
\mathcal{L}=\pi \dot{\phi}-\frac{1}{2} \pi^{2}+\pi \lambda-\frac{1}{2} \lambda^{2}-\frac{1}{2} \phi^{\prime 2}-\lambda \phi^{\prime},
$$


Now, as mentioned above, we have to make the canonical transformations

$$
\phi=\eta+\sigma \quad \text { and } \quad \pi=\eta^{\prime}-\sigma^{\prime},
$$

which is defined as a dynamical decomposition. Note that $\phi$ is already a chiral field. Thus, in this way, this canonical transformation will allow us to know the Srivastava chiral boson exactly. Hence, substituting Eq. (20) into Eq. (19), we have, as a result,

$$
\mathcal{L}_{D D}=\eta^{\prime} \dot{\eta}-\eta^{\prime 2}-\sigma^{\prime} \dot{\sigma}-\sigma^{\prime 2}-2 \lambda \sigma^{\prime}-\frac{1}{2} \lambda^{2} .
$$

Again, solving the equations of motion for the $\lambda$ field, we have $\lambda=-2 \sigma^{\prime}$, and, substituting again,

$$
\mathcal{L}_{D D}=\eta^{\prime} \dot{\eta}-\eta^{\prime 2}-\left(\sigma^{\prime} \dot{\sigma}-\sigma^{\prime 2}\right) .
$$

We can clearly see that this action represents two FJ chiral bosons, each one with the same chirality. This is caused by the fact that the Lagrange multiplier has acquired dynamics because of the linear constraint form. In fact, we are demonstrating that Eq. (17) has two degrees of freedom, represented in Eq. (21) by $\eta$ and $\sigma$. This is different from Siegel's approach, where $\lambda$ is a pure gauge degree of freedom. This result corroborates the one found by Bazeia [32], analyzing the linear constraint chiral boson quantum mechanics. We can say that both particles in Eq. (21) act like a GuptaBleuler pair, so that each chiral excitation destroys the other and the Hilbert space is composed of vacuum. This result confirms the one found in Ref. [33].

Hence, in the soldering process of the SCB, each FJ chiral boson interacts with its opposite chiral partner, so that the final result represents a scalar field. We can also observe that the linear constraint formulation of the chiral boson does not contain a Hull noton [38], a nonmover field that cancels out the anomaly of the Siegel model (an alternative fermionic noton was introduced in Ref. [39]), which is expected since the SCB is not gauge invariant.

Result (21) contradicts the result obtained in Ref. [23] in the following way. There, first, a final action was built, composed of the Srivastava action plus a WZ term with an arbitrary parameter. The Lorentz invariance fixed the parameter in two possible values, which caused two different WZ terms. Hence one of the actions obtained, after a kind of chiral decomposition, is shown to have two FJ particles of opposite chiralities, analogously to what occurs with the usual CSM [40]. In addition, the final Lagrangian obtained contains auxiliary fields, the so-called BF fields (details in [24]) used to construct the WZ term [41]. The authors conclude that the WZ term is constructed to add an additional degree of freedom to the theory in the form of an antichiral boson. On the other hand, we can see what is really happening through a careful analysis of the two WZ terms introduced in Ref. [23]. It is not difficult to see the first WZ term defined in [23], i.e.,

$$
\mathcal{L}_{W Z}^{(1)}=-\frac{1}{2}\left(\dot{\theta}^{2}+3 \theta^{\prime 2}\right)-\lambda\left(\dot{\theta}+\theta^{\prime}\right)-\frac{1}{2} \lambda^{2},
$$

where $\theta$ is the $\mathrm{BF}$ field, and, once integrated in the $\lambda$ field, a chiral boson is recovered. In addition, if one takes the second WZ term introduced in Ref. [23],

$$
\mathcal{L}_{W Z}^{(2)}=-\dot{\theta} \theta^{\prime}-\theta^{\prime 2}-\lambda\left(\dot{\theta}+\theta^{\prime}\right)-\frac{1}{2} \lambda^{2},
$$

and again performs the integration $\lambda$, one obtains nothing but the Lagrangian density of the free scalar boson. This result signalizes that the WZ term obtained by Miao et al. [23] is already composed of two opposite FJ particles. Obviously this introduces the degree of freedom, already there in the WZ term, but it does not change the physics of the SCB model, since, as we saw above, this model is composed of vacuum.

Analyzing the interference aspects, we can again apply the soldering formalism; now, however, we do it using two actions like Eq. (21), i.e.,

$$
\begin{aligned}
& \mathcal{L}_{1}=\dot{\eta} \eta^{\prime}-\eta^{\prime 2}-\left(\dot{\sigma} \sigma^{\prime}-\sigma^{\prime 2}\right), \\
& \mathcal{L}_{2}=-\dot{\xi} \xi^{\prime}-\xi^{\prime 2}-\left(-\dot{\omega} \omega^{\prime}-\omega^{\prime 2}\right),
\end{aligned}
$$

where $\eta, \sigma, \xi$, and $\omega$ are all $\mathrm{FJ}$ particles. We can see in Eqs. (24) and (25) that the fields $(\eta, \xi)$ and $(\sigma, \omega)$ form opposite chirality particle pairs.

Performing the soldering procedure, one can easily see that the result is

$$
\mathcal{L}=\frac{1}{2}\left(\partial_{\mu} \Psi\right)-\frac{1}{2}\left(\partial_{\mu} \Lambda\right)
$$

where $\Psi=\eta-\xi$ and $\Lambda=\sigma-\omega$. This, and not Eq. (16), is the expected result, since we know that the SCB has a vacuumlike spectrum. The soldering procedure in Eq. (26) discloses the same behavior as shown in Eq. (21).

Result (26) is quite different from the one shown in Eq. (16). Since it is well known that the soldering of two opposite FJ chiral bosons is a massless scalar field, we should expect that the fusion of two SCB's would be two opposite scalar fields with the final vacuumlike spectrum. This difference can be explained [31]: we note that now, in each action of Eqs. (24) and (25), we have two fields, i.e., the action can be separated into two different sectors, representing FJ particles with the same chirality. Thus, in the interference process (soldering), each sector of each action interferes with its opposite partner. To obtain Eq. (16), note that we have only one sector in each action. In the interference process we have lost information about the other sector, such as destructive interference. This does not occur in Eq. (26).

\section{CONCLUSIONS}

It is well known that the SCB has consistency problems. In this work we have used the soldering formalism to show that the on-shell interference of two Srivastava chiral bosons resulted in a scalar field. The other aspect of this result is that the soldering method recovers the consistency of the SCB model, i.e., the fusion of opposite chiralities of the model results in a consistent theory. This contradicts the conclusion published in the literature, which asserts that it is impossible to apply the soldering procedure to the SCB due to the in- 
equivalence of this model in relation to Siegel's and Floreanini and Jackiw's models.

This motivated us to explore the model, promoting a canonical transformation in the specific form of a dynamical decomposition, which permitted us to decompose the action into its dynamical parts. This procedure showed us that the $\mathrm{SCB}$ is in fact formed by two Floreanini-Jackiw chiral bosons of the same chiralities. Again, the contradiction with the current literature is evident, since one well-known publication affirmed that the WZ term introduced a new degree of freedom to the theory, resulting in two Floreanini-Jackiw chiral bosons of opposite chiralities: a chiral boson and an antichiral boson. This is not really true, since we saw that in fact the WZ term used consists of two degrees of freedom, i.e., two FJ opposite chiral particles. Thus, one can say that it is obvious that this WZ term should introduce new degrees of freedom, because it is comprised of the fields that appeared. With each SCB composed of two fields, after fusion through the soldering formalism we obtained two scalar fields with a negative signal between them. This result shows that the spectrum of the soldered action is vacuumlike.

\section{ACKNOWLEDGMENTS}

The authors would like to thank C. Wotzasek and S. J. Gates, Jr. for valuable discussions. E.M.C.A. was financially supported by Fundação de Amparo à Pesquisa do Estado de São Paulo (FAPESP). This work was partially supported by Conselho Nacional de Pesquisa e Desenvolvimento (CNPq). FAPESP and CNPq are Brazilian research agencies.
[1] W. Siegel, Nucl. Phys. B238, 307 (1984).

[2] R. Floreanini and R. Jackiw, Phys. Rev. Lett. 59, 1873 (1987).

[3] N. Marcus and J. Schwarz, Phys. Lett. 115B, 111 (1982); D. J. Gross, J. A. Harvey, E. Martinec, and R. Rhom, Phys. Rev. Lett. 54, 502 (1985).

[4] M. Gomes, V. Kurak, V. O. Rivelles, and A. J. da Silva, Phys. Rev. D 38, 1344 (1988).

[5] X. G. Wen, Phys. Rev. Lett. 64, 2206 (1990); Phys. Rev. B 41, 12838 (1990); M. Stone, ibid. 42, 212 (1990).

[6] H. O. Girotti, M. Gomes, V. Kurak, V. O. Rivelles, and A. J. da Silva, Phys. Rev. Lett. 60, 1913 (1988).

[7] M. Stone, Illinois report, ILL - (TH) - 89-23, 1989; Phys. Rev. Lett. 63, 731 (1989); Nucl. Phys. B327, 399 (1989); D. Depireux, S. J. Gates, Jr., and Q.-Han Park, Phys. Lett. B 224, 363 (1989); E. Witten, Commun. Math. Phys. 144, 189 (1992).

[8] A. A. Kirilov, Elements of the Representation Theory (Nauka, Moscow, 1972); A. Alekseev, L. Faddeev, and S. Shatashvili, J. Geom. Phys. 5, 391 (1989); A. Alekseev and S. Shatashvili, Nucl. Phys. B323, 719 (1989).

[9] E. Witten, Commun. Math. Phys. 92, 455 (1984).

[10] H. O. Girotti, M. Gomes, V. O. Rivelles, and A. J. da Silva, Phys. Rev. D 39, 3792 (1989).

[11] P. P. Srivastava, Phys. Rev. Lett. 63, 2791 (1989); Phys. Lett. B 234, 93 (1990).

[12] K. Harada, Phys. Rev. Lett. 65, 267 (1990).

[13] H. O. Girotti, M. Gomes, and V. O. Rivelles, Phys. Rev. D 45, R3329 (1992).

[14] Y. G. Miao, J. Phys. G 17, L177 (1991); D. S. Kulshreshtha and H. J. W. Müller-Kirsten, Phys. Rev. D 45, R393 (1992); M. A. Zhongshui, Commun. Theor. Phys. 28, 217 (1997); Y. G. Miao, ibid. 19, 125 (1993); R. Amorim and J. Barcelos-Neto, Z. Phys. C 68, 513 (1995).

[15] Y. G. Miao and H. J. W. Müller-Kirsten, Phys. Rev. D 62, 045014 (2000).

[16] A. A. Deriglazov, Theor. Math. Phys. 92, 748 (1992).

[17] K. Harada, Int. J. Mod. Phys. A 6, 3399 (1991).

[18] E. M. C. Abreu, R. Banerjee, and C. Wotzasek, Nucl. Phys. B509, 519 (1998).
[19] J. Schwinger, Phys. Rev. 128, 2425 (1962).

[20] R. Jackiw and R. Rajaraman, Phys. Rev. Lett. 54, 1219 (1985).

[21] J. Wess and B. Zumino, Phys. Lett. B 234, 93 (1990).

[22] L. D. Faddeev and S. Shatashvili, Phys. Lett. 167B, 225 (1986); K. Harada and I. Tsutsui, Phys. Lett. B 183, 311 (1987); O. Babelon, F. A. Schaposnik, and C. M. Viallet, ibid. 177, 385 (1986).

[23] Y. G. Miao, J. G. Zhou, and Y. Y. Liu, Phys. Lett. B 323, 169 (1994).

[24] I. Batalin and E. S. Fradkin, Nucl. Phys. B279, 514 (1987); Phys. Lett. B 180, 157 (1986).

[25] L. D. Faddeev, Phys. Lett. 145B, 81 (1984); M. Kobayashi, K. Seo, and A. Sugamoto, Nucl. Phys. B273, 687 (1986).

[26] W. H. Kye, W. T. Kim, and J. K. Kim, Phys. Lett. B 268, 59 (1991); A. de Souza Dutra, ibid. 286, 285 (1992).

[27] Y. G. Miao and C. H. Chen, Commun. Theor. Phys. 30, 317 (1998).

[28] R. Amorim, A. Das, and C. Wotzasek, Phys. Rev. D 53, 5810 (1996).

[29] G. Dunne, R. Jackiw, and C. Trugenberger, Phys. Rev. D 41, 661 (1990).

[30] R. Banerjee and S. Ghosh, Phys. Lett. B 482, 302 (2000).

[31] E. M. C. Abreu and C. Wotzasek, Phys. Rev. D 58, 101701(R) (1998).

[32] D. Bazeia, Mod. Phys. Lett. A 6, 1147 (1991).

[33] C. Wotzasek, Mod. Phys. Lett. A 8, 2509 (1993).

[34] For a review, see C. Wotzasek, hep-th/9806005.

[35] E. M. C. Abreu, A. Ilha, C. Neves, and C. Wotzasek, Phys. Rev. D 61, 025014 (2000).

[36] R. Amorim and A. Das, Phys. Rev. D 54, 4177 (1996).

[37] H. O. Girotti, H. J. Rothe, and K. D. Rothe, Phys. Rev. D 33, 514 (1986).

[38] C. M. Hull, Phys. Lett. B 206, 234 (1988); 212, 437 (1988).

[39] D. Depireux, S. Gates, and B. Radak, Phys. Lett. B 236, 408 (1990).

[40] A. de Souza Dutra, Phys. Lett. B 286, 285 (1992).

[41] T. Fujiwara, Y. Igarashi, and J. Kubo, Nucl. Phys. B341, 695 (1990). 